\title{
Identification of two CiGADs from Caragana intermedia and their transcriptional responses to abiotic stresses and exogenous abscisic acid
}

\author{
Jing Ji ${ }^{1}$, Lingyu Zheng ${ }^{1}$, Jianyun Yue ${ }^{1}$, Xiamei Yao ${ }^{1}$, Ermei Chang ${ }^{1}$, Tiantian Xie ${ }^{1}$, Nan Deng ${ }^{1}$, Lanzhen

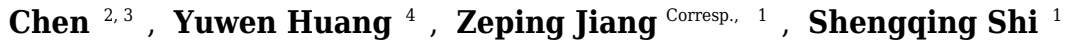 \\ ${ }^{1}$ State Key Laboratory of Tree Genetics and Breeding, Chinese Academy of Forestry, Research Institute of Forestry, Beijing, China \\ 2 Institute of Apicultural Research, Chinese Academy of Agricultural Sciences, Beijing, China \\ 3 Risk Assessment Laboratory for Bee Products, Quality and Safety of Ministry of Agriculture, Beijing, China \\ 4 The High School Affiliated to Renmin University of China, Beijing, China \\ Corresponding Author: Zeping Jiang \\ Email address: jiangzp@caf.ac.cn
}

Background. Glutamate decarboxylase (GAD), as a key enzyme in the $\gamma$-aminobutyric acid (GABA) shunt, catalyzes the decarboxylation of L-glutamate to form GABA. This pathway has attracted much interest because of its roles in carbon and nitrogen metabolism, stress responses, and signaling in higher plants. The aim of this study was to isolate and characterize genes encoding GADs from Caragana intermedia, an important nitrogen-fixing leguminous shrub.

Methods. Two full-length cDNAs encoding GADs (designated as CiGAD1 and CiGAD2) were isolated and characterized. Multiple alignment and phylogenetic analyses were conducted to evaluate their structures and identities to each other and to homologs in other plants. Tissue expression analyses were conducted to evaluate their transcriptional responses to stress $\left(\mathrm{NaCl}, \mathrm{ZnSO}_{4}, \mathrm{CdCl}_{2}\right.$, high/low temperature, and dehydration) and exogenous abscisic acid.

Results. The CiGADs contained the conserved PLP domain and calmodulin (CaM)-binding domain in the C-terminal region. The phylogenetic analysis showed that they were more closely related to the GADs of soybean, another legume, than to GADs of other model plants. According to Southern blotting analysis, CiGAD1 had one copy and CiGAD2-related genes were present as two copies in C. intermedia. In the tissue expression analyses, there were much higher transcript levels of CiGAD2 than CiGAD1 in bark, suggesting that CiGAD2 might play a role in secondary growth of woody plants. Several stress treatments $\left(\mathrm{NaCl}, \mathrm{ZnSO}_{4}, \mathrm{CdCl}_{2}\right.$, high/low temperature, and dehydration) significantly increased the transcript levels of both CiGADs, except for CiGAD2 under Cd stress. The CiGAD1 transcript levels strongly increased in response to $\mathrm{Zn}$ stress (74.3-fold increase in roots) and heat stress (218.1-fold increase in leaves). The transcript levels of both CiGADs significantly increased as GABA accumulated during a 24-h salt treatment. Abscisic acid was involved in regulating the expression of these two CiGADs under salt stress.

Discussion. This study showed that two CiGADs cloned from C. intermedia are closely related to homologs in another legume, soybean. CiGAD2 expression was much higher than that of CiGAD1 in bark, indicating that CiGAD2 might participate in the process of secondary growth in woody plants. Multiple stresses, interestingly, showed that $\mathrm{Zn}$ and heat stresses had the strongest effects on CiGAD1 expression, suggesting that CiGAD1 plays important roles in the responses to $\mathrm{Zn}$ and heat stresses. Additionally, these two genes might be involved in ABA dependent pathway during stress. This result provides important information about the role of GADs in woody plants' responses to environmental stresses. 
1 Identification of two CiGADs from Caragana intermedia and their transcriptional responses to

2 abiotic stresses and exogenous abscisic acid

3 Jing $\mathrm{Ji}^{1 *}$, Lingyu Zheng ${ }^{{ }^{*}}$, Jianyun Yue ${ }^{1}$, Xiamei Yao ${ }^{1}$, Ermei Chang ${ }^{1}$, Tiantian Xie, Nan Deng ${ }^{1}$,

4 Lanzhen Chen ${ }^{2,3}$, Yuwen Huang ${ }^{4}$, Zeping Jiang ${ }^{1 \#}$, Shengqing Shi ${ }^{1 \#}$

$5 \quad{ }^{1}$ State Key Laboratory of Tree Genetics and Breeding, Research Institute of Forestry, Chinese

6 Academy of Forestry, Beijing, China

$7 \quad$ Institute of Apicultural Research, Chinese Academy of Agricultural Sciences, Beijing, China

8 3Risk Assessment Laboratory for Bee Products, Quality and Safety of Ministry of Agriculture,

9 Beijing, China

$10{ }^{4}$ The High School Affiliated to Renmin University of China, Beijing, China

$12+$ Corresponding author: Zeping Jiang

13 Email address: jiangzp@caf.ac.cn;

$14{ }^{\dagger}$ Corresponding author: Shengqing Shi

15 Email address: shi.shengqing@caf.ac.cn

$16{ }^{*}$ Contribute equally to this work.

17 \# Co-corresponding authors. 


\section{ABSTRACT}

Background. Glutamate decarboxylase (GAD), as a key enzyme in the $\gamma$-aminobutyric acid (GABA) shunt, catalyzes the decarboxylation of L-glutamate to form GABA. This pathway has attracted much interest because of its roles in carbon and nitrogen metabolism, stress responses, and signaling in higher plants. The aim of this study was to isolate and characterize genes encoding GADs from Caragana intermedia, an important nitrogen-fixing leguminous shrub.

Methods. Two full-length cDNAs encoding GADs (designated as CiGAD1 and CiGAD2) were isolated and characterized. Multiple alignment and phylogenetic analyses were conducted to evaluate their structures and identities to each other and to homologs in other plants. Tissue expression analyses were conducted to evaluate their transcriptional responses to stress $(\mathrm{NaCl}$, $\mathrm{ZnSO}_{4}, \mathrm{CdCl}_{2}$, high/low temperature, and dehydration) and exogenous abscisic acid.

Results. The CiGADs contained the conserved PLP domain and calmodulin (CaM)-binding domain in the C-terminal region. The phylogenetic analysis showed that they were more closely related to the GADs of soybean, another legume, than to GADs of other model plants. According to Southern blotting analysis, CiGAD1 had one copy and CiGAD2-related genes were present as two copies in $C$. intermedia. In the tissue expression analyses, there were much higher transcript levels of CiGAD2 than CiGAD1 in bark, suggesting that CiGAD2 might play a role in secondary growth of woody plants. Several stress treatments $\left(\mathrm{NaCl}, \mathrm{ZnSO}_{4}, \mathrm{CdCl}_{2}\right.$, high/low temperature, and dehydration) significantly increased the transcript levels of both CiGADs, except for CiGAD2 under Cd stress. The CiGAD1 transcript levels strongly increased in response to $\mathrm{Zn}$ stress (74.3-fold increase in roots) and heat stress (218.1-fold increase in leaves). The transcript levels of both CiGADs significantly increased as GABA accumulated during a 24-h salt treatment. Abscisic acid was involved in regulating the expression of these two CiGADs under salt stress.

Discussion. This study showed that two CiGADs cloned from $C$. intermedia are closely related to homologs in another legume, soybean. CiGAD2 expression was much higher than that of CiGAD1 in bark, indicating that CiGAD2 might participate in the process of secondary growth in woody plants. Multiple stresses, interestingly, showed that $\mathrm{Zn}$ and heat stresses had the strongest effects on CiGAD1 expression, suggesting that CiGAD1 plays important roles in the responses to $\mathrm{Zn}$ and heat stresses. Additionally, these two genes might be involved in $\mathrm{ABA}$ dependent pathway during stress. This result provides important information about the role of GADs in woody plants' responses to environmental stresses.

\section{Introduction}

Y-Aminobutyric acid (GABA) is a non-protein amino acid present in animals, plants, and other organisms (Batushansky et al., 2014; Gilliham \& Tyerman, 2016; Michaeli \& Fromm., 2015; Shelp et al., 2012a,b). It functions as a major inhibitory neurotransmitter in the brain tissues of mammals (Granger et al., 2016; Robel \& Sontheimer, 2015). In plants, GABA plays roles in 
diverse processes including the carbon:nitrogen balance, signaling, regulation of redox status, development, stress responses (Batushansky et al., 2014, 2015; Michaeli \& Fromm, 2015, Molina-Rueda et al., 2015; Shi et al., 2010; Yu et al., 2014). Recent studies have indicated that GABA is required for proper growth during exposure to abiotic stresses such as salt (Renault et al., 2013), drought (Mekonnen et al., 2016), low temperature (Aghdam et al., 2016), $\mathrm{Zn}^{2+}$ (Daş et al., 2016), and cadmium (Sun et al., 2010). Additionally, exogenous GABA has been shown to regulate the expression of genes such as BnNrt2 in Brassica napus (Beuve et al., 2004), 14-3-3 in Arabidopsis thaliana (Lancien \& Roberts, 2006), SAMDC (S-adenosylmethionine decarboxylase) in Cucumis melo (Wang et al., 2014), and to control gene transcription in Caragana intermedia (Shi et al., 2010) and $A$. thaliana (Batushansky et al., 2014). The metabolite GABA has been shown to play roles in a diverse range of cellular processes ranging from neuronal inhibition in animals to pollen-tube development in plants (Michaeli \& Fromm, 2015). Consequently, many researchers have focused on the production of GABA, and in particular, the role of glutamate decarboxylase (GAD), which is the unique pyridoxal enzyme catalyzing the $\alpha$-decarboxylation of L-glutamate to form GABA (Bouché \& Fromm, 2004; Shelp et al., 2012a,b).

The pathway of glutamate decarboxylation catalyzed by GAD is widely distributed in organisms (Liu et al., 2014), and is considered to be the main pathway of GABA production in plants (Shelp et al., 2012a). Genes encoding GAD enzymes have been successfully identified in many herbaceous plants such as rice (Akama et al., 2001), Arabidopsis (Bouché et al., 2004), maize (Zhuang et al., 2010), and Panax ginseng (Lee et al., 2010) and in some woody plants, such as pine (Molina-Rueda et al., 2010), apple (Trobacher et al., 2013), citrus (Liu et al, 2014), and Tea (Mei et al., 2016). Bioinformatics analyses and experimental characterizations have shown that plant GADs contain a calmodulin (CaM)-binding domain that is generally responsible for the cytosolic decarboxylation of glutamate to GABA (Shelp et al., 2012a), although one functional OsGAD2 without a $\mathrm{Ca}^{2+} /$ calmodulin domain has been isolated from rice (Akama et al., 2001). Several studies have demonstrated spatiotemporal differences in the expressions of $G A D$ gene family member, although most GAD families studied to date have fewer than nine members (Shelp et al., 2012a). For example, AtGAD1 is mainly expressed in Arabidopsis roots (Miyashita \& Good, 2008), while ZmGAD1 is expressed in the leaves, stems, and roots of maize (Zhuang et al., 2010). In rice, OsGAD1 is mainly expressed in seeds but OsGAD2 is mainly expressed in roots (Akama et al., 2001). In citrus, CSGAD1 is predominantly expressed flowers but CsGAD2 is predominantly expressed in fruit (Liu et al., 2014). Interestingly PpGAD expression was shown to be correlated with vascular differentiation in pine seedlings (Molina-Rueda et al., 2010). Additionally, environmental stimuli were shown to induce the expression of ZmGAD1 in maize (Zhuang et al., 2010) and PgGAD in P. ginseng (Lee et al., 2010). These studies indicate that plant GADs might play important roles in plant development and stress responses. However, few studies have focused on the expression of GADs in woody plants under environmental stresses.

Our previous studies showed that salt stress induced significant expression of EST sequences (Supplementary T1) homologous to plant GAD genes in the desert legume shrub, $C$. intermedia. 
We also showed that exogenous GABA can enhance the expression of genes, including those related to hormones and reactive oxygen species (ROS), which involved the stress responses of this shrub (Shi et al., 2010). Therefore, in the present study, we cloned two full-length cDNAs, CiGAD1 and CIGAD2, from C. intermedia based on known EST sequences using the rapid amplification of cDNA ends (RACE) technique. We analyzed changes in their transcript levels in response to different stress factors and exogenous abscisic acid ( $A B A)$. These results allowed us to explore the relationship between GAD expression and GABA accumulation, which will increase our understanding of the roles of GABA in stress adaptation in plants.

\section{Materials and Methods}

\subsection{Plant materials and treatments}

Seeds of $C$. intermedia were collected from a desert habitat in Inner Mongolia, China. Seedlings were cultivated as described by Shi et al. (2010). Briefly, seeds were washed and then germinated in sand. Three-day-old seedlings (radical length, 30-40 mm) were selected and cultivated with water in pots in a growth chamber under a 14-h light/10-h dark photoperiod and a day/night temperature of $23 / 18^{\circ} \mathrm{C}$. The seedlings were grown for $2-3$ weeks, and the water was refreshed every 2 days. Subsequently, the seedlings were subjected to the following stress treatments: (1) $\mathrm{NaCl}(200 \mathrm{mM}), \mathrm{ZnSO}_{4}(100 \mu \mathrm{M}), \mathrm{CdCl}_{2}(500 \mu \mathrm{M})$, high temperature (40 ${ }^{\circ} \mathrm{C}$ ), low temperature $\left(4{ }^{\circ} \mathrm{C}\right)$, or dehydration (for $3 \mathrm{~h}$ ); (2) $\mathrm{NaCl}(200 \mathrm{mM}$ ) for different durations $(0,3,6,12$, and $24 \mathrm{~h})$; and (3) exogenous $\operatorname{ABA}(0,0.5,1,10,100$, and $200 \mu \mathrm{M})$ combined with the $200 \mathrm{mM} \mathrm{NaCl}$ treatment for 0,3 , and $24 \mathrm{~h}$. Roots and leaves were harvested, frozen immediately in liquid nitrogen, and then stored at $-80^{\circ} \mathrm{C}$. Also, different tissues including roots, stems, leaves, seeds, testae, and bark were collected from mature individuals growing at the Chinese Academy of Forestry, Beijing, frozen immediately in liquid nitrogen, and then stored at $-80^{\circ} \mathrm{C}$. Every experimental treatment had three biological replicates.

\subsection{Cloning, sequencing, and bioinformatic analysis}

Total RNA was extracted from pooled roots and leaves with Trizol reagent (Invitrogen, Carlsbad,

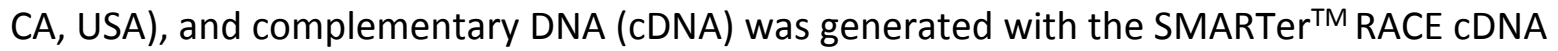
amplification kit (Clontech, Palo Alto, CA, USA) according to the manufacturer's instructions. Then, 5' and 3' RACE were performed using nested gene-specific primers (Supplementary table S1). The obtained amplification products were cloned into the pMD19-D vector (Takara, Dalian, China) and sequenced. Then, the two full-length sequences were assembled by DNAMAN 6.0. CiGAD1 and CIGAD2 have been deposited in GenBank under the accession numbers KU586714 and KU586715, respectively.

The two full-length cDNA sequences of CiGAD1 and CiGAD2 were analyzed with ORF Finder (https://www.ncbi.nlm.nih.gov/orffinder/), and the isoelectric point (pl) and molecular weight (MW) of the predicted protein were estimated using ExPASy (http://web.expasy.org/compute_pi/). Sequences for the protein homology analysis were obtained from phytozome (https://phytozome.jgi.doe.gov/pz/portal.html), and aligned using 
134 DNAMAN 8.0. The phylogenetic tree was constructed using MEGA 6.06 software by the maximum-likelihood algorithm with 1000 bootstrap replicates.

136

137

138

139

140

141

142

143

144

145

146

147

148

149

150

151

152

\subsection{Southern blot analysis}

Genomic DNA was isolated from leaves of $C$. intermedia using CTAB method (Porebski et al., 1991), and $20 \mu \mathrm{g}$ DNAs were digested with BamH I and EcoR V, respectively. The obtaining fragments were separated by electrophoresis through a 1.0\% agarose, then DIG-labeled DNA probes of CIGAD1 and CiGAD2 were hybridized followed the protocol of DIG-High Prime DNA Labeling and Detection Starter Kit I (Roche Applied Science, Mannheim, Germany). Briefly, DIGlabeled DNA probe was prehybridized at $60^{\circ} \mathrm{C}$ for $2 \mathrm{~h}$ and then hybridized $37^{\circ} \mathrm{C}$ for $12 \mathrm{~h}$. Following hybridization, the blot was washed twice in $2 \times \mathrm{SSC}$ containing $0.1 \%$ SDS at $25^{\circ} \mathrm{C}$ for 5 min and was washed twice in $0.1 \times \mathrm{SSC}$ containing $0.1 \%$ SDS at $50{ }^{\circ} \mathrm{C}$ for $15 \mathrm{~min}$. The immunodetection of the DIG-labeled probe was performed with 1:10000 anti DIG-AP. The blot was exposed to X-ray film (AGFA, Germany). DNA probes were amplified from genomic DNA by using the primers designed in the sequenced DNA fragments based on the cDNA sequences of CIGAD1 and CIGAD2, while the probe for CIGAD1 was mainly in the region from 1076-1370 bp in the full-text cDNA, and the probe for CiGAD2 was mainly in the region from 639-1031 bp in the full-text cDNA. Primers listed in Supplementary table S1 and probe sequences listed in Supplementary T3.

\subsection{Assay of GAD activity}

About $0.1 \mathrm{~g}$ sample (root, stem, leaf, seed, or bark) was ground into a powder in liquid nitrogen and transferred to a 2-mL centrifuge tube. Then, $1 \mathrm{~mL}$ pre-cooled extraction buffer $[150 \mathrm{mM}$ potassium phosphate, $5 \mathrm{mM}$ EDTA, $1 \mathrm{mM}$ magnesium chloride, $0.5 \%(\mathrm{w} / \mathrm{v})$ polyvinylpyrrolidone, $3 \mathrm{mM}$ 2-mercaptoethanol, $0.2 \mathrm{mM}$ PLP (pyridoxal 5'-phosphate), 10\% (v/v) glycerol, $1 \mathrm{mM}$ phenylmethylsulfonyl fluoride, $\mathrm{pH} \mathrm{5.8]} \mathrm{was} \mathrm{added,} \mathrm{and} \mathrm{the} \mathrm{homogenate} \mathrm{was} \mathrm{mixed} \mathrm{well.} \mathrm{After}$ 15 min incubation on ice, the homogenate was centrifuged at $10,000 \mathrm{~g}$ for $15 \mathrm{~min}$ at $4{ }^{\circ} \mathrm{C}$. The supernatant was collected into a 2-mL volumetric flask and the volume was adjusted to $1 \mathrm{~mL}$. This was used as the crude enzyme solution (CES; Liu et al., 2014).

To measure GAD activity, the assay mixture $(1 \mathrm{~mL})$ contained $0.4 \mathrm{~mL} 0.1 \%$ sodium glutamate, $0.2 \mathrm{~mL} 0.25 \mathrm{mM}$ PLP, and $0.4 \mathrm{~mL}$ CES. After mixing well, the reaction mixture was incubated at $40{ }^{\circ} \mathrm{C}$ for $5 \mathrm{~h}$ and then boiled for $5 \mathrm{~min}$ to end the reaction. Sodium glutamate was replaced by $0.4 \mathrm{~mL}$ distilled water in the blank control. After cooling, the absorbance of the mixture was measured at $640 \mathrm{~nm}$ using a Multi-Mode Detection Platform (SpectraMax Paradigm, Molecular Devices Co., Sunnyvale, CA, USA). A GABA standard curve was constructed and used to calculate the GABA content in the samples based on absorption value, as described by Yang et al. (2006). And protein contents were tested by Bradford method (Kruger, 1994) with bovine serum albumin as standard. One unit (U) was defined as the amount ( $\mu \mathrm{mol}$ ) of GABA produced by the action of one $\mathrm{mg}$ protein per hour. All determinations were representative of three biological experiments and four technical replicates. 


\subsection{Determination of GABA}

The plant tissues were ground to a fine powder in liquid nitrogen, and about $0.1 \mathrm{~g}$ of the frozen homogenate was extracted using methanol and lanthanum chloride, and then centrifuged at $13000 \mathrm{~g}$ for $5 \mathrm{~min}$, and $0.8 \mathrm{ml}$ of the supernatant was transferred to a new Eppendorf tube. The $160 \mu \mathrm{l}$ of $1 \mathrm{M} \mathrm{KOH}$ was added, and centrifugation as before. The $100 \mu \mathrm{l}$ resulting supernatant was used in the spectrophotometric GABA determination (Zhang \& Bown 1997). The assay mixture $(300 \mu \mathrm{l})$ also contained $75 \mathrm{mM}$ potassium pyrophosphate $(\mathrm{pH} 8.6), 3.3 \mathrm{mM} 2-$ mercaptoethanol, $1.25 \mathrm{mM} \beta-\mathrm{NADP}^{+}, 5 \mathrm{mM}$ 2-ketoglutarate and 0.02 units of GABase (Sigma). The increase of OD340 $\mathrm{nm}$ was recorded using 96-well microplate reader. The amount of GABA was calculated according to external calibration curve of GABA (Renault et al., 2010). Values shown were representative of three biological experiments and four technical replicates.

\section{6 qRT-PCR}

Total RNAs were extracted from a pool of roots and leaves with Trizol reagent (Invitrogen), and then cDNAs were synthesized with $0.5 \mu$ g RNAs using the Prime Script ${ }^{T M}$ RT reagent kit (Perfect Real Time; Takara) according to the manufacturer's instructions. Specific RT-PCR primers (Supplementary table S2) were designed to have melting temperatures of $60{ }^{\circ} \mathrm{C}$ and amplicon lengths of 150-200 bp using Primer3 software (http://primer3.ut.ee/). Real-time qRT-PCR was performed in quadruplicate using the SYBR Premix Ex Taq ${ }^{\text {TM }}$ II kit (Takara) on a Roche lightCycler 480 (Roche Applied Sciences, Penzberg, Germany) according to the manufacturer's instructions.

The $2^{-\triangle \mathrm{Ct}}$ method was adopted to analyze the qRT-PCR result, and $\triangle \mathrm{Ct}=\mathrm{Ct}_{\text {target }}{ }^{-\mathrm{Ct}} \mathrm{EF1 \alpha}$. Values shown were representative of three biological experiments.

\subsection{Statistical analysis}

Data were evaluated by Duncan's multiple test in SPSS v.19. Differences were considered significant at $\mathrm{P}<0.05$.

\section{Results}

\subsection{Isolation of CiGADs and bioinformatics analyses}

Two full-length cDNAs of glutamate decarboxylase (GAD) genes were isolated from $C$. intermedia by 5'- and 3'-RACE, as shown in Supplementary fig. S1, and designated as CiGAD1 (GenBank ID: KU586714) and CiGAD2 (GenBank ID: KU586715). Bioinformatics analyses showed that CiGAD1 had a 1922-bp full-length mRNA sequence containing a 1521-bp open reading frame (ORF), a 107-bp 5'puntranslated region (UTR), and a 294-bp 3'-UTR; and that CiGAD2 had a 1797-bp full-length mRNA sequence containing a 1797-bp ORF, a 60-bp 5'-UTR, and a 237-bp 3'-UTR. The alignment showed that the nucleotide identity between CIGAD1 and CiGAD2 was 69.0\%. Theoretically, CiGAD1 encodes a 57.3-kDa peptide consisting of 506 amino acids with the theoretical isoelectric point (pl) of 5.5, and CiGAD2 encodes a 56.1-kDa peptide consisting of 499 amino acids with the theoretical isoelectric point $(\mathrm{pl})$ of 5.6. 
208 The deduced amino acid sequences of the two CiGADs were aligned with GAD family members

209 from three typical model plants; Arabidopsis, Glycine, and Populus (Supplementary fig. S2). The

210 amino acid identity between CiGAD1 and CiGAD2 was 71.4\%. CiGAD1 shared 88.5\%, 79.9\% and

$21179.4 \%$ amino acid identity with Glycine GAD5, Arabidopsis GAD1, and Populus GAD1,

212 respectively. CsGAD2 shared 83.1\%, 75.0\%, and 72.1\% amino acid identity with Glycine GAD2,

213 Populus GAD5, and Arabidopsis GAD5, respectively. Both CiGAD proteins contained two

214 conserved domains: a PLP-binding domain in the middle region and a CaM-binding domain at

215 the carboxyl terminus (Fig. 1). The ML tree showed that CiGAD1 was located close to GmGAD5,

216 GmGAD1, and GmGAD3; while CiGAD2 was in a completely different clade, close to GmGAD2,

217 GmGAD4, PtGAD5, and AtGAD5 (Fig. 2). These findings indicated that CiGAD proteins from $C$.

218 intermedia, a salt- and drought-resistant desert legume shrub, had higher homologies with GAD

219 homologs in soybean, another well-known legume.

220 We also performed a southern blot analysis to estimate the copy number of two GAD genes in

221 C. intermedia. The result showed one band in BamH I or EcoR I restriction digest lane of CiGAD1

222 (Fig. 3A) and two bands in the BamH I or EcoRI restriction digest lane of CiGAD2 (Fig. 3B),

223 respectively. This confirmed that CiGAD1 had one copy, and CiGAD2 -related genes were

224 present as two copies in the genome of $C$. intermedia.

\subsection{Tissue-specific expression of two CiGADs}

The expression of two CiGADs showed different patterns in various issues including roots, stems, leaves, seeds, testae, and bark from mature individuals (Fig. 4A). The transcript levels of CiGAD1 were lower than those of CiGAD2 in roots, seeds, testae, and bark, but the opposite pattern was observed in other tissues. The two genes showed contrasting trends in mRNA abundance in most tissues. The highest transcript level of CiGAD1 was in testae, followed by leaves and stems, while it was almost undetectable in roots and bark. In contrast, CiGAD2 showed the highest transcript level in the bark (37.9-fold that of CiGAD1 in the bark), followed by testae and roots (1.9 and 21.3 fold higher, respectively, than that of CiGAD1). The GAD activity was highest in testaes (9.9 U), followed by seeds (3.2 U). But its activity was lower, and similar, among the other tested tissues (1.1-1.9 U) (Fig. 4B).

\subsection{Stress-specific expression of two CiGADs}

To investigate whether the two CiGADs were expressed differently in response to various stress conditions, total RNA was extracted from roots and leaves of young seedlings under several different stress treatments. Both CiGADs showed dramatic increases in their transcript levels in roots and leaves in response to stress (Fig. 5A, B). Under Zn stress, the transcript level of CiGAD1 increased by 74.3-fold and that of CiGAD2 increased by 6.3-fold, compared with their respective levels in control roots. CiGAD1 showed 3.4-11.3 fold increases in its transcript levels in roots under dehydration, $\mathrm{Cd}, \mathrm{Na}$, and low/high temperature stresses. CiGAD2 transcript levels showed 1.0-5.5 fold increases in roots under all of the stresses except Cd stress (Fig. 5A). In leaves, the transcript levels of CIGAD1 and CiGAD2 strongly increased in response to high temperature, by 218.1-fold and 114.2-fold, respectively, compared with their respective levels 
247 in the control leaves (Fig. 5B). The transcript levels of CiGAD1 and CiGAD2 increased by 20.6-

248 fold and 36.0-fold, respectively, under $\mathrm{NaCl}$ stress, and by 19.7-fold and 21.8-fold, respectively, 249 under low temperature stress. Under $\mathrm{Zn}, \mathrm{Cd}$, and dehydration stress, CiGAD1 expression

250 increased by $4.4-8.5$ fold, and CiGAD2 expression increased by $1.5-5.2$ fold. These results 251 indicated that the transcript levels of CiGAD1 and CiGAD2 increased to varying degrees in 252 response to various stresses.

To analyze the expression of the two CiGADs under stress in more detail, seedlings were treated with $\mathrm{NaCl}$ for different times, as shown in Fig. 6 . The transcript levels of CiGAD1 and CiGAD2 significantly increased as the duration of the $\mathrm{NaCl}$ treatment extended, although there were some fluctuations in their transcript levels in leaves at $12 \mathrm{~h}$ and in roots at $6 \mathrm{~h}$ (Fig. 6A, B). In roots, the transcript levels of CIGAD1 and CiGAD2 had increased by 20.8-fold and 16.4-fold, respectively, after $6 \mathrm{~h}$ of salt stress, and by 41.2 -fold and 12.0 -fold, respectively, after $24 \mathrm{~h}$ of salt stress, compared with their respective levels at $0 \mathrm{~h}$ (Fig. 6A). However, in the leaves, CiGAD1 and CiGAD2 transcript levels remained relatively stable except at $6 \mathrm{~h}$ of salt stress, when they were increased by 20.6-23.7 fold, and 11.5-13.8 fold, respectively, compared with their respective levels at $0 \mathrm{~h}$ (Fig. 6B). Further analyses showed that CiGAD1 was more strongly induced than was CiGAD2 as the $\mathrm{NaCl}$ treatment extended. Corresponding to the expression of CiGADs, there was a considerable increase in endogenous GABA with increasing duration of the $\mathrm{NaCl}$ treatment (Fig. $6 \mathrm{C}$ ). The GABA content in roots increased rapidly, to 5.9-fold its initial level after $3 \mathrm{~h}$ of $\mathrm{NaCl}$ stress, and to more than 10-fold its initial level at 12 and $24 \mathrm{~h}$ of $\mathrm{NaCl}$ stress. In the leaves, the GABA content slightly increased after $3 \mathrm{~h}$ of $\mathrm{NaCl}$ stress, and markedly increased to $4.7-5.6$ fold its initial level by $6 \mathrm{~h}$ of $\mathrm{NaCl}$ stress. These findings suggested that there was a positive correlation between the expression of CiGAD genes and GABA accumulation during $\mathrm{NaCl}$ stress.

\subsection{ABA regulated CiGADs expression under $\mathrm{NaCl}$ stress}

Treatment of $\mathrm{NaCl}$-stressed seedlings with exogenous ABA significantly affected the expression of both CiGADs, and the changes in expression differed between the two genes (Fig. 7). In the roots, both CiGADs showed gradually increasing transcript levels with increasing concentrations of exogenous $A B A$ at $3 \mathrm{~h}$. However, CiGAD1 showed a 3.8-fold increase in its transcript levels in response to $0.5 \mu \mathrm{M} A B A$, similar to the increase in response to $200 \mu \mathrm{M} A B A$. The two genes showed almost opposite trends in expression in the roots at $24 \mathrm{~h}$ of treatment with $A B A$; the transcript levels of CiGAD1 decreased with increasing $A B A$ concentrations, but were still much higher than the transcript levels of CiGAD2 (Fig. 7A). In the leaves, however, after $3 \mathrm{~h}$ of $A B A$ treatment, low concentrations of exogenous $A B A(0.5$ and $1.0 \mu \mathrm{M})$ inhibited the expression of both CiGADs, but higher concentrations of ABA induced the expression of both genes. After 24 $h$ of treatment with $A B A, 0.5 \mu \mathrm{M} A B A$ considerably increased the expression levels of both genes to more than 5.6-fold that in the control ( $0 \mu \mathrm{M} A B A)$, but $A B A$ at other concentrations drastically reduced the transcript levels of both genes, except for CiGAD1 at $10 \mu \mathrm{M}$ ABA (Fig. 7B). These results demonstrated that, even at micromolar concentrations, $A B A$ participates in regulating CiGAD1 and CiGAD2 transcription during salt stress. 


\section{Discussion}

Much attention has been paid to the roles of GABA in the development and stress responses of plants (Batushansky et al., 2014, 2015; Michaeli and Fromm, 2015, Molina-Rueda et al., 2015; Shi et al., 2010). Because GAD is a key enzyme regulating GABA production and the GABA shunt pathway (Michaeli and Fromm, 2015; Shimajiri et al., 2013). In this study, two GAD genes (CiGAD1 and CiGAD2) were cloned based on specific EST sequences (Supplementary T1) identified in our previous work on the molecular response of $C$. intermedia to salt stress. These two genes had ORFs encoding 498- and 494-amino acid residues. Homology analyses showed that these two CiGADs exhibited relatively high similarity $(72.1 \%-88.5 \%)$ to GADs from three model plants: soybean, Arabidopsis, and poplar, so CiGADs were classified into the GAD family (Supplementary fig. S2). The CaM/Ca ${ }^{2+}$-dependent GADs conserved the putative CaM-binding domain in plants, such as Arabidopsis (Turano et al., 1998), tomato (Gallego et al., 1995), and $P$. ginseng (Lee at al., 2010). But some GADs did not include CaM-binding domain, such as rice GAD2 (Akama et al., 2001). Sequence analyses confirmed that both of the CiGADs included a conserved PLP domain and a CaM-binding domain (Fig. 1; Trobacher et al., 2013), but whether both CiGADs were indeed activated via CaM or not need a further research (Mei et al., 2016). A phylogenetic analysis indicated that CIGAD1 and CiGAD2 were much more closely related to $G A D$ genes in soybean than to GAD genes in the woody plant poplar (Fig. 2). Southern blot analysis confirmed that CiGAD1 had one copy and CiGAD2 -related genes were present as two copies in C. intermedia (Fig. 3). However, both AtGAD1 and AtGAD2 are single copy genes in Arabidopsis (Turano et al., 1998), and single copy of GmGAD1 is also observed in soybean (Matsuyama et al., 2009). But there are at least two copy of the PgGAD in P. ginseng (Lee at al., 2010).These results indicate that the copy number of $G A D$ genes depends on each plant.

The cloning of CiGADs genes will be useful for further research on their functions during plant development and stress responses. As mentioned in the Introduction, GADs are expressed in most tissues, but different GADs show different spatial and temporal expression patterns (Miyashita \& Good, 2008; Akama et al., 2001; Liu et al., 2014). Our results showed that CiGAD1 was mainly expressed in the stems, leaves, and testae; whereas CiGAD2 was expressed at high levels in bark and was also expressed in the roots and testae (Fig. 4A). The expression patterns indicated that CIGAD2 might participate in vascular differentiation, like PpGAD in maritime pine (Molina-Rueda et al., 2010). Molina-Rueda et al. (2015) also reported that vascular development was closely linked to GABA production corresponding to PpGAD expression. Because the GAD activities were higher in testae and seeds, CiGAD1 and CiGAD2 may also play a role in plant reproduction like GADs in citrus (Liu et al., 2014) and apple (Trobacher et al. 2013).

Several studies have shown that plant GADs expression responds differently to various abiotic stresses in herbaceous plants (Zhuang et al., 2010; Lee et al., 2010). Our previous findings showed that exogenous GABA can regulate the molecular responses of $C$. intermedia during salt stress via its roles in regulating signaling (reactive oxygen species, ethylene, and ABA-producing enzymes $\mathrm{RBOH} / \mathrm{ACO} / \mathrm{ABA} 2$ ) and nitrogen metabolism (nitrate transporter, arginase, nodulin) 
327 (Shi et al., 2010). These results prompted us to analyze the expression patterns of the two identified CiGADs in response to various stresses and exogenous $A B A$, which orchestrates the interaction between biotic and abiotic stresses via signaling pathways (Atkinson \& Urwin, 2012). When Caragana seedlings were subjected to short-term $(3 \mathrm{~h})$ stress treatments including salinity $(\mathrm{Na})$, heavy metals $(\mathrm{Zn}$ and $\mathrm{Cd})$, heat $\left(40^{\circ} \mathrm{C}\right)$, cold $\left(4^{\circ} \mathrm{C}\right)$, and drought ( $3 \mathrm{~h}$ dehydration), the transcript levels of CiGAD1 and CiGAD2 increased in the roots and leaves (Fig. 3), possibly as a result of an increase in $\mathrm{Ca}^{2+}$ induced by each stress treatment (Akihiro et al., 2008). The only exception was that CiGAD2 transcript levels did not increase in response to $\mathrm{Cd}^{2+}$ (Fig. 4A, B), which is hypothesized to displace metal cofactors (such as $\mathrm{Zn}^{2+}$ ) from proteins or compete with $\mathrm{Ca}^{2+}$ to bind to $\mathrm{Ca}^{2+}$-binding proteins (Stohs \& Bagchi, 1995). The salt stress treatment induced CiGAD1 and CiGAD2 expression more strongly in leaves (20.6-36.0 fold) than in roots (4.4-5.5 fold) (Fig. 5A, B). For comparison, PgGAD expression in $P$. ginseng increased 3 -fold after $8 \mathrm{~h}$ of salt treatment (Lee et al., 2010). This result indicated that the two CiGADs might play a crucial role in the salt stress response in leaves.

In terms of heavy metal stress responses, the $\mathrm{Zn}$ treatment significantly increased expression of both CiGADs in leaves and roots, and induced a substantial increase in CiGAD1 expression (a 74.0-fold increase) in roots (Fig. 5A), whereas $\mathrm{Cd}$ had opposite effects on the two genes, inducing an increase in CiGAD1 expression (4.4-6.0 fold), but no change or a decrease in CiGAD2 expression (Fig. 5A, B). An analysis of the Arabidopsis transcriptome showed that AtGAD4 transcript levels increased 8.4-fold under a $50 \mu \mathrm{M} \mathrm{Cd}^{2+}$ treatment (Weber et al., 2006). A surprising result in this study was that heat and cold stresses strongly induced expression of CiGAD1 and CiGAD2, especially in leaves; the transcript levels of these genes increased by 218.1-fold and 114.2-fold, respectively, in response to heat, and by 19.7-fold and 21.8-fold, respectively, in response to cold (Fig. 5B). For comparison, PgGAD expression in $P$. ginseng increased by 3-5 fold after 8 -h heat and cold treatments (Lee et al., 2010). Next, we examined changes in the transcript levels of two CiGADs during a 24-h salt treatment, and observed constant increases in their expression (Fig. 6A, B) consistent with GABA accumulation (Fig. 6C). The present result suggested that these two CiGADs in Caragana were involved in multiple stress responses, especially the responses to $\mathrm{Zn}$ and heat stress.

In plants, the phytohormone $A B A$ is a key endogenous signaling molecule in the responses to various stresses. Understanding ABA-signaling is essential for improving plant performance in the future (Atkinson \& Urwin, 2012; Raghavendra et al., 2010). In our previous study, we found that exogenous GABA induced the expression of the ABA-biosynthetic gene $A B A 2$ in Caragana under salt stress (Shi et al., 2010). Exogenous ABA has also been shown to enhance GAD activity and increase the GABA content in fava bean (Yang et al., 2016), and to induce the expression of ZmGAD1, which harbors ABA-related cis-elements in its promoter (Zhuang et al., 2010). In the present study, we found that exogenous ABA increased the transcript levels of CiGAD1 and CiGAD2 at an early stage ( $3 \mathrm{~h}$ ) of the salt treatment (Fig.7A, B); while after $24 \mathrm{~h}$ of salt treatment, the transcript levels of both genes were reduced in the roots of ABA-treated plants, but strongly increased in the leaves of plants treated with $0.5 \mu \mathrm{M}$ ABA (Fig. 7A, B). These 
different transcriptional responses of CIGAD1 and CiGAD2 to exogenous ABA may be because of differences in ABREs in their promoter regions (Liu et al., 2014).

In conclusion, the results of this study showed that two CiGADs cloned from Caragana are closely related to homologs in another legume, soybean. The transcript levels of CiGAD2 were much higher than those of CIGAD1 in bark, suggesting that CiGAD2 might play a role in secondary growth of woody plants. The GAD activities were higher in testae and seeds than other tissues, CiGADs may also play a role in plant reproduction. Multiple stresses significantly affected the transcript levels of both CiGADs. Interestingly, $\mathrm{Zn}$ and heat stresses had the strongest effects on CiGAD1 expression, indicating that CiGAD1 plays important roles in the responses to $\mathrm{Zn}$ and heat stresses. Our results also suggested that $A B A$ is involved in regulating these two CiGADs during stress in Caragana, an important nitrogen-fixing legume shrub. These results will be useful for further research on the role of GADs in carbon and nitrogen metabolism and in signal transduction during the responses to environmental stresses.

\section{References}

Aghdam MS, Naderi R, Jannatizadeh A, Babalar M, Sarcheshmeh MAA, Faradonbe MZ. 2016. Impact of exogenous GABA treatments on endogenous GABA metabolism in anthurium cut flowers in response to postharvest chilling temperature. Plant Physiology and Biochemistry 106: 11-15. DOI: 10.1016/j.plaphy.2016.04.045.

Akama K, Akihiro T, Kitagawa M, Takaiwa F. 2001. Rice (Oryza sativa) contains a novel isoform of glutamate decarboxylase that lacks an authentic calmodulin-binding domain at the Cterminus. Biochimica et Biophysica Acta (BBA)-Gene Structure and Expression 1522: 143150. DOI: 10.1016/S0167-4781(01)00324-4.

Akihiro T, Koike S, Tani R, Tominaga T, Watanabe S, lijima Y, Aoki K, Shibata D, Ashihara H, Matsukura C. 2008. Biochemical mechanism on GABA accumulation during fruit development in tomato. Plant and Cell Physiology 49: 1378-1389. DOI: 10.1093/pcp/pcn113.

Atkinson NJ, Urwin PE. 2012. The interaction of plant biotic and abiotic stresses: from genes to the field. Journal of Experimental Botany 63: 3523-3543. DOI: 10.1093/jxb/ers100.

Batushansky A, Kirma M, Grillich N, Pham PA, Rentsch D, Galili G, Fernie AR, Fait A. 2015. The transporter GAT1 plays an important role in GABA-mediated carbon-nitrogen interactions in Arabidopsis. Frontiers in Plant Science 6. DOI: 10.3389/fpls.2015.00785

Batushansky A, Kirma M, Grillich N, Toubiana D, Pham PA, Balbo I, Fromm H, Galili G, Fernie AR, Fait A. 2014. Combined transcriptomics and metabolomics of Arabidopsis thaliana seedlings exposed to exogenous GABA suggest its role in plants is predominantly metabolic. Molecular Plant 7: 1065-1068. DOI: 10.1093/mp/ssu017.

Beuve N, Rispail N, Laine P, CLIQUET JB, Ourry A, Le Deunff E. 2004. Putative role of $\gamma$ aminobutyric acid (GABA) as a long-distance signal in up-regulation of nitrate uptake in 
404

405

406

407

408

409

410

411

412

Brassica napus L. Plant, Cell and Environment 27: 1035-1046. DOI: 10.1111/j.13653040.2004.01208.x.

Bouché N, Fait A, Zik M, Fromm H. 2004. The root-specific glutamate decarboxylase (GAD1) is essential for sustaining GABA levels in Arabidopsis. Plant Molecular Biology 55: 315-325. DOI: 10.1007/s11103-004-0650-z.

Bouche N, Fromm H. 2004. GABA in plants: just a metabolite? Trends in Plant Science 9: 110115. DOI: 10.1016/j.tplants.2004.01.006.

Daş ZA, Dimlioğlu G, Bor M, Özdemir F. 2016. Zinc induced activation of GABA-shunt in tobacco (Nicotiana tabaccum L.). Environmental and Experimental Botany 122: 78-84. DOI: 10.1016/j.envexpbot.2015.09.006.

Gallego PP, Whotton L, Picton S, Grierson D, Gray JE. 1995. A role for glutamate decarboxylase during tomato ripening: the characterisation of a cDNA encoding a putative glutamate decarboxylase with a calmodulin-binding site. Plant Molecular Biology 27: 1143-1151. DOI: 10.1007/BF00020887

Gilliham M, Tyerman SD. 2016. Linking metabolism to membrane signaling: the GABA-malate connection. Trends in Plant Science 21: 295-301. DOI: 10.1016/j.tplants.2015.11.011.

Granger AJ, Mulder N, Saunders A, Sabatini BL. 2016. Cotransmission of acetylcholine and GABA. Neuropharmacology 100: 40-46. DOI: 10.1016/j.neuropharm.2015.07.031.

Lancien M, Roberts MR. 2006. Regulation of Arabidopsis thaliana 14-3-3 gene expression by $\gamma^{-}$ aminobutyric acid. Plant, Cell and Environment 29: 1430-1436. DOI: 10.1111/j.13653040.2006.01526.x.

Lee JH, Kim YJ, Jeong DY, Sathiyaraj G, Pulla RK, Shim JS, In JG, Yang DC. 2010. Isolation and characterization of a glutamate decarboxylase (GAD) gene and their differential expression in response to abiotic stresses from Panax ginseng C. A. Meyer. Molecular Biology Reports 37: 3455-3463. DOI: 10.1007/s11033-009-9937-0.

Matsuyama A, Yoshimura K, Shimizu C, Murano Y, Takeuchi H, Ishimoto M. 2009. Characterization of glutamate decarboxylase mediating $\mathrm{Y}$-amino butyric acid increase in the early germination stage of soybean (Glycine max [L.] Merr). Journal of Bioscience and Bioengineering 107: 538-543. DOI: 10.1016/j.jbiosc.2009.01.012.

Liu X, Hu XM, Jin LF, Shi CY, Liu YZ, Peng SA. 2014. Identification and transcript analysis of two glutamate decarboxylase genes, CSGAD1 and CSGAD2, reveal the strong relationship between CSGAD1 and citrate utilization in citrus fruit. Molecular Biology Reports 41: 62536262. DOI: 10.1007/s11033-014-3506-x.

Mekonnen DW, Flügge UI, Ludewig F. 2016. Gamma-aminobutyric acid depletion affects stomata closure and drought tolerance of Arabidopsis thaliana. Plant Science 245: 25-34. DOI: 10.1016/j.plantsci.2016.01.005. 
Mei X, Chen Y, Zhang L, Fu X, Wei Q, Grierson D, Zhou Y, Huang Y, Dong F, Yang Z. 2016. Dual mechanisms regulating glutamate decarboxylases and accumulation of gammaaminobutyric acid in tea (Camellia sinensis) leaves exposed to multiple stresses. Scientific Reports 6. DOI: 10.1038/srep23685

Michaeli S, Fromm H. 2015. Closing the loop on the GABA shunt in plants: are GABA metabolism and signaling entwined? Frontiers in Plant Science 6. DOI: 10.3389/fpls.2015.00419.

Miyashita Y, Good AG. 2008. Contribution of the GABA shunt to hypoxia-induced alanine accumulation in roots of Arabidopsis thaliana. Plant and Cell Physiology 49: 92-102. DOI: $10.1093 / \mathrm{pcp} / \mathrm{pcm} 171$.

Molina-Rueda JJ, Pascual MB, Cánovas FM, Gallardo F. 2010. Characterization and developmental expression of a glutamate decarboxylase from maritime pine. Planta 232: 1471-1483. DOI: 10.1007/s00425-010-1268-9.

Molina-Rueda JJ, Pascual MB, Pissarra J, Gallardo F. 2015. A putative role for $\gamma$-aminobutyric acid (GABA) in vascular development in pine seedlings. Planta 241: 257-267. DOI: 10.1007/s00425-014-2157-4.

Kruger NJ. 1994. The Bradford method for protein quantitation. Basic Protein and Peptide Protocols 9-15.

Porebski S, Bailey LG, Baum BR. 1997. Modification of a CTAB DNA extraction protocol for plants containing high polysaccharide and polyphenol components. Plant Molecular Biology Reporter 15: 8-15. DOI: 10.1007/BF02672076.

Raghavendra AS, Gonugunta VK, Christmann A, Grill E. 2010. ABA perception and signalling. Trends in Plant Science 15: 395-401. DOI: 10.1016/j.tplants.2010.04.006.

Renault H, Roussel V, El Amrani A, Arzel M, Renault D, Bouchereau A, Deleu C. 2010. The Arabidopsis pop2-1 mutant reveals the involvement of GABA transaminase in salt stress tolerance. BMC Plant Biology, 10: 20. DOI: 10.1186/1471-2229-10-20.

Renault H, El Amrani A, Berger A, Mouille G, Soubigou-Taconnat L, Bouchereau A, Deleu C. 2013. $\gamma$-Aminobutyric acid transaminase deficiency impairs central carbon metabolism and leads to cell wall defects during salt stress in Arabidopsis roots. Plant, Cell and Environment 36: 1009-1018. DOI: 10.1111/pce.12033.

Robel S, Sontheimer H. 2016. Glia as drivers of abnormal neuronal activity. Nature Neuroscience 19: 28-33. DOI: 10.1038/nn.4184.

Shelp BJ, Bozzo GG, Trobacher CP, Chiu G, Bajwa VS. 2012a. Strategies and tools for studying the metabolism and function of $\gamma$-aminobutyrate in plants. I. Pathway structure. Botany 90 : 651-668. DOI: 10.1139/b2012-041.

Shelp BJ, Bozzo GG, Zarei A, Simpson JP, Trobacher CP, Allan WL. 2012b. Strategies and tools for 
studying the metabolism and function of $\mathrm{\gamma}$-aminobutyrate in plants. II. Integrated analysis. Botany 90: 781-793. DOI: 10.1139/b2012-030.

Shi SQ, Shi Z, Jiang ZP, Qi LW, Sun XM, Li CX, Liu JF, Xiao WF, Zhang SG. 2010. Effects of exogenous GABA on gene expression of Caragana intermedia roots under $\mathrm{NaCl}$ stress: regulatory roles for $\mathrm{H}_{2} \mathrm{O}_{2}$ and ethylene production. Plant, Cell and Environment 33: 149162. DOI: 10.1111/j.1365-3040.2009.02065.x.

Shimajiri Y, Oonishi T, Ozaki K, Kainou K, Akama K. 2013. Genetic manipulation of the $\mathrm{Y}^{-}$ aminobutyric acid (GABA) shunt in rice: overexpression of truncated glutamate decarboxylase (GAD2) and knockdown of $\gamma$-aminobutyric acid transaminase (GABA-T) lead to sustained and high levels of GABA accumulation in rice kernels. Plant Biotechnology Journal 11: 594-604. DOI: 10.1111/pbi.12050.

Stohs S, Bagchi D. 1995. Oxidative mechanisms in the toxicity of metal ions. Free Radical Biology and Medicine 18: 321-336. DOI: 10.1016/0891-5849(94)00159-H.

Sulieman S. 2011. Does GABA increase the efficiency of symbiotic $\mathrm{N}_{2}$ fixation in legumes? Plant Signaling and Behavior 6: 32-36. DOI: 10.4161/psb.6.1.14318.

Sun X, Zhang J, Zhang H, Ni Y, Zhang Q, Chen J, Guan Y. 2010. The responses of Arabidopsis thaliana to cadmium exposure explored via metabolite profiling. Chemosphere 78: 840-845. DOI: 10.1016/j.chemosphere.

Trobacher CP, Zarei A, Liu J, Clark SM, Bozzo GG, Shelp BJ. 2013. Calmodulin-dependent and calmodulin-independent glutamate decarboxylases in apple fruit. BMC Plant Biology 13: 1. DOI: 10.1186/1471-2229-13-144.

Turano FJ, Fang T. K. 1998. Characterization of two glutamate decarboxylase cDNA clones from Arabidopsis. Plant Physiology 117: 1411-1421. DOI: 10. 1104/pp.117.4.1411.

Wang C, Fan L, Gao H, Wu X, Li J, Lv G, Gong B. 2014. Polyamine biosynthesis and degradation are modulated by exogenous gamma-aminobutyric acid in root-zone hypoxia-stressed melon roots. Plant Physiology and Biochemistry 82: 17-26. DOI: 10.1016/j.plaphy.2014.04.018.

Weber M, Trampczynska A, Clemens S. 2006. Comparative transcriptome analysis of toxic metal responses in Arabidopsis thaliana and the $\mathrm{Cd}^{2+}$-hypertolerant facultative metallophyte Arabidopsis halleri. Plant, Cell and Environment 29: 950-963. DOI: 10.1111/j.13653040.2005.01479.x.

Yang $\mathrm{R}$, Hui $\mathrm{Q}$, Gu Z. 2016. Effects of $\mathrm{ABA}$ and $\mathrm{CaCl}_{2}$ on $\mathrm{GABA}$ accumulation in fava bean germinating under hypoxia- $\mathrm{NaCl}$ stress. Bioscience, Biotechnology, and Biochemistry 80 : 540-546. DOI: 10.1080/09168451.2015.1116923.

Yu GH, Zou J, Feng J, Peng XB, Wu JY, Wu YL, Palanivelu R, Sun MX. 2014. Exogenous Yaminobutyric acid (GABA) affects pollen tube growth via modulating putative $\mathrm{Ca}^{2+}$ 
511 permeable membrane channels and is coupled to negative regulation on glutamate

512 decarboxylase. Journal of Experimental Botany 65: 3235-3248. DOI: 10.1093/jxb/eru171.

513 Zhang G, Bown AW. 1997. The rapid determination of $\mathrm{\gamma}$-aminobutyric acid. Phytochemistry 44:

514 1007-1009. DOI: 10.1016/S0031-9422(96)00626-7.

515 Zhuang Y, Ren G, He C, Li X, Meng Q, Zhu C, Wang R, Zhang J. 2010. Cloning and characterization 516 of a maize cDNA encoding glutamate decarboxylase. Plant Molecular Biology Reporter 28:

517 620-626. DOI: 10.1007/s11105-010-0191-3. 


\section{Figure 1}

Alignment of the deduced amino acid sequence of two CiGADs in Caragana intermedia with the deduced amino acid residues of GADs in Arabidopsis thaliana, Populus trichocarpa, Glycine max.

Accession number: AtGAD1 (AT5G17330), AtGAD2 (AT1G65960), AtGAD3 (AT2G02000), AtGAD4 (AT2G02010), AtGAD5 (AT3G17760), GmGAD1 (Glyma02g40840), GmGAD2 (Glyma09g29900), GmGAD3 (Glyma14g39170), GmGAD4 (Glyma16g34450), GmGAD5 (Glyma18g04940), PtGAD1 (Potri.T059200), PtGAD2 (Potri.004G075200), PtGAD3 (Potri.004G075300), PtGAD4 (Potri.010G100500), PtGAD4.1 (Potri.008G141100), and PtGAD5 (Potri.012G039000). The abbreviation of gene names are as follows: At, Arabidopsis thaliana; Pt, Populus trichocarpa; Gm, Glycine max. Identical and similar amino acid residues were shown in black or pink, respectively. The pyridoxal-5-phosphate binding domain of the GADs was underlined with a thin line, and the CaM binding domain was underlined with a thick line; Trp (W), the important site for in vitro binding to CaM, was indicated by an asterisk. 


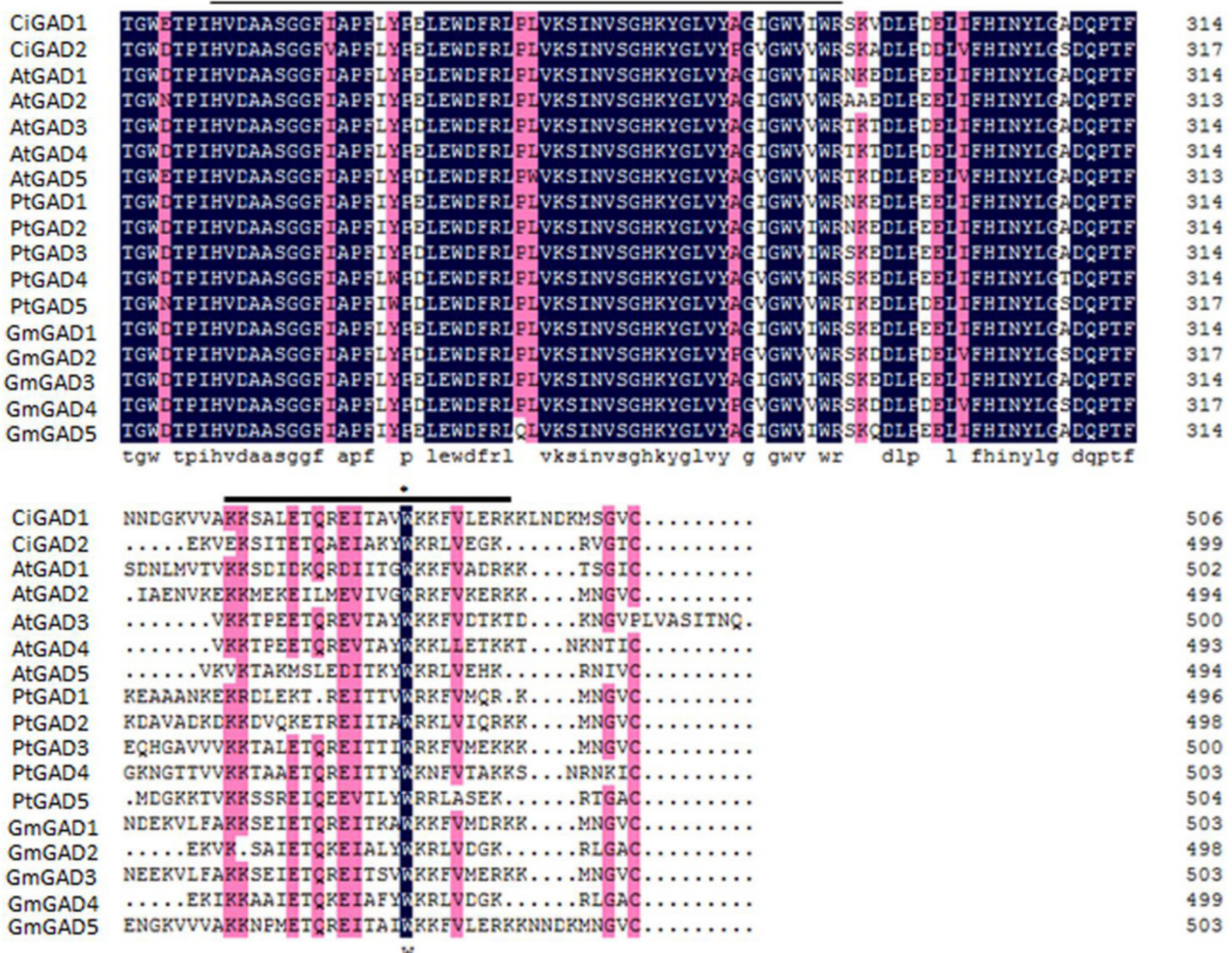


Figure 2

Phylogenetic analysis of GAD sequences from Caragana intermedia and other three model plants Arabidopsis thaliana, Populus trichocarpa, Glycine max.

The consensus tree was obtained by the Maximum-Likelihood method in MEGA 6.06. A bootstrap analysis of 1,000 replicates was performed. Accession numbers were as shown in Legend of Figure 1. The candidate GADs were marked with black triangle.

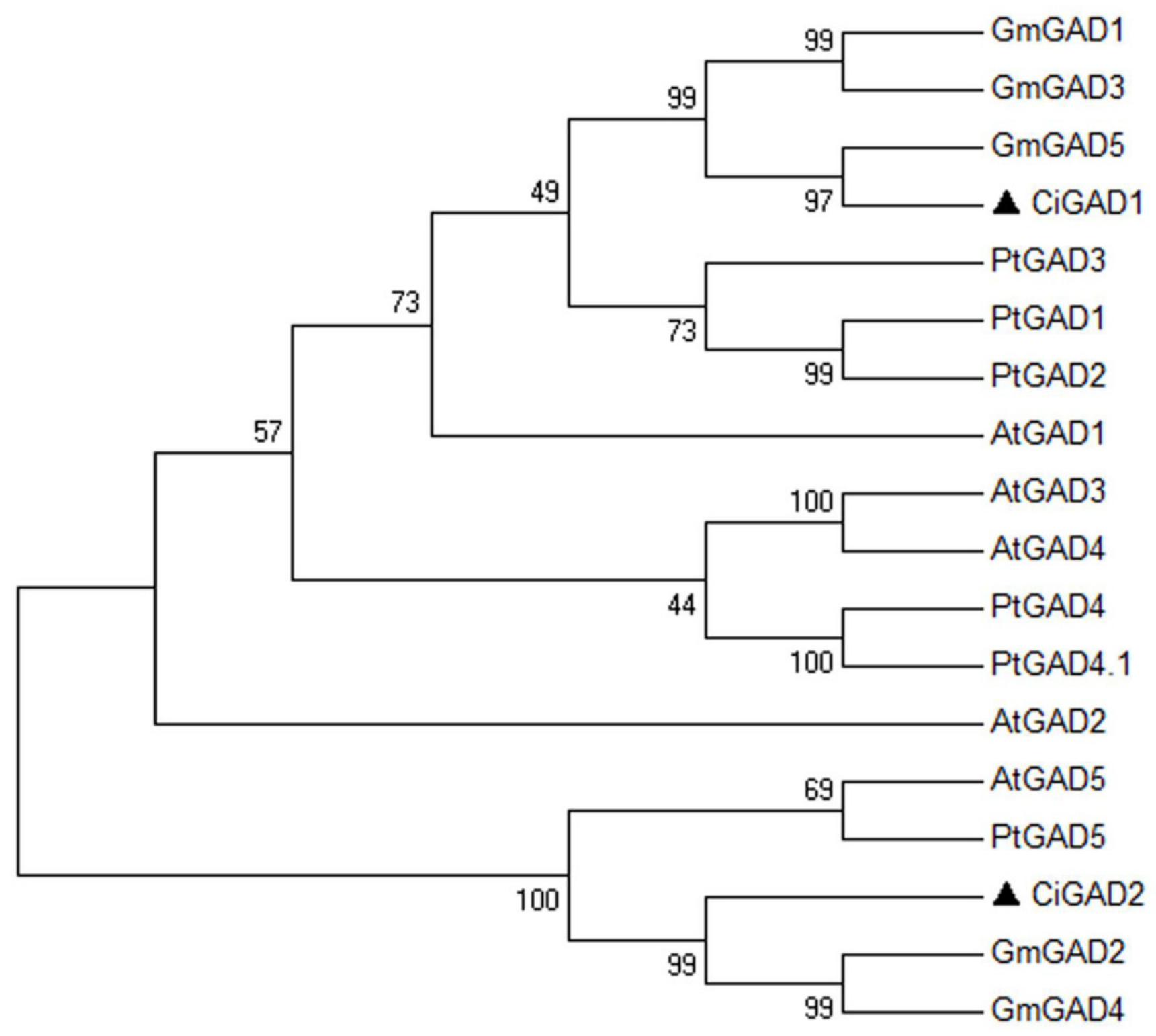


Figure 3

Southern blotting analysis of CiGAD1 (A) and CiGAD2 (B)

Lane 1: BamH I; Lane 2: EcoR V. Red arrows were targeted bands; M: 10,000 bp DNA marker; blank: DNA probe.

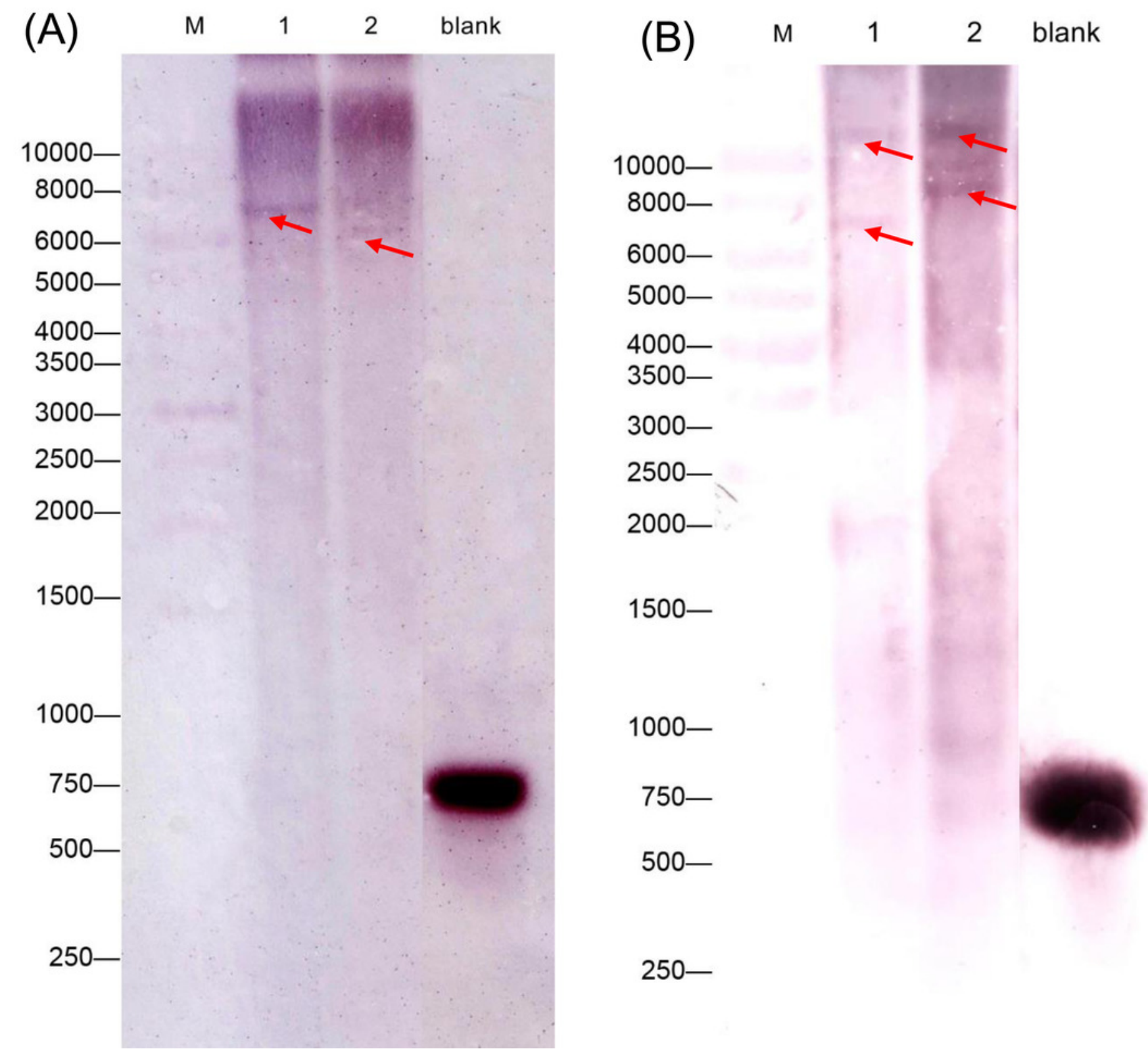




\section{Figure 4}

Expression of two CiGADs (A) and total GAD activity (B) in different tissues of the mature Caragana intermedia, including roots, stems, leaves, seeds, testae and bark.

The means and standard errors were calculated from three biological replicates. Different lower-case letters between any two sampling points indicate signification difference at $P<$ 0.05 by Duncan's test. 
(A)

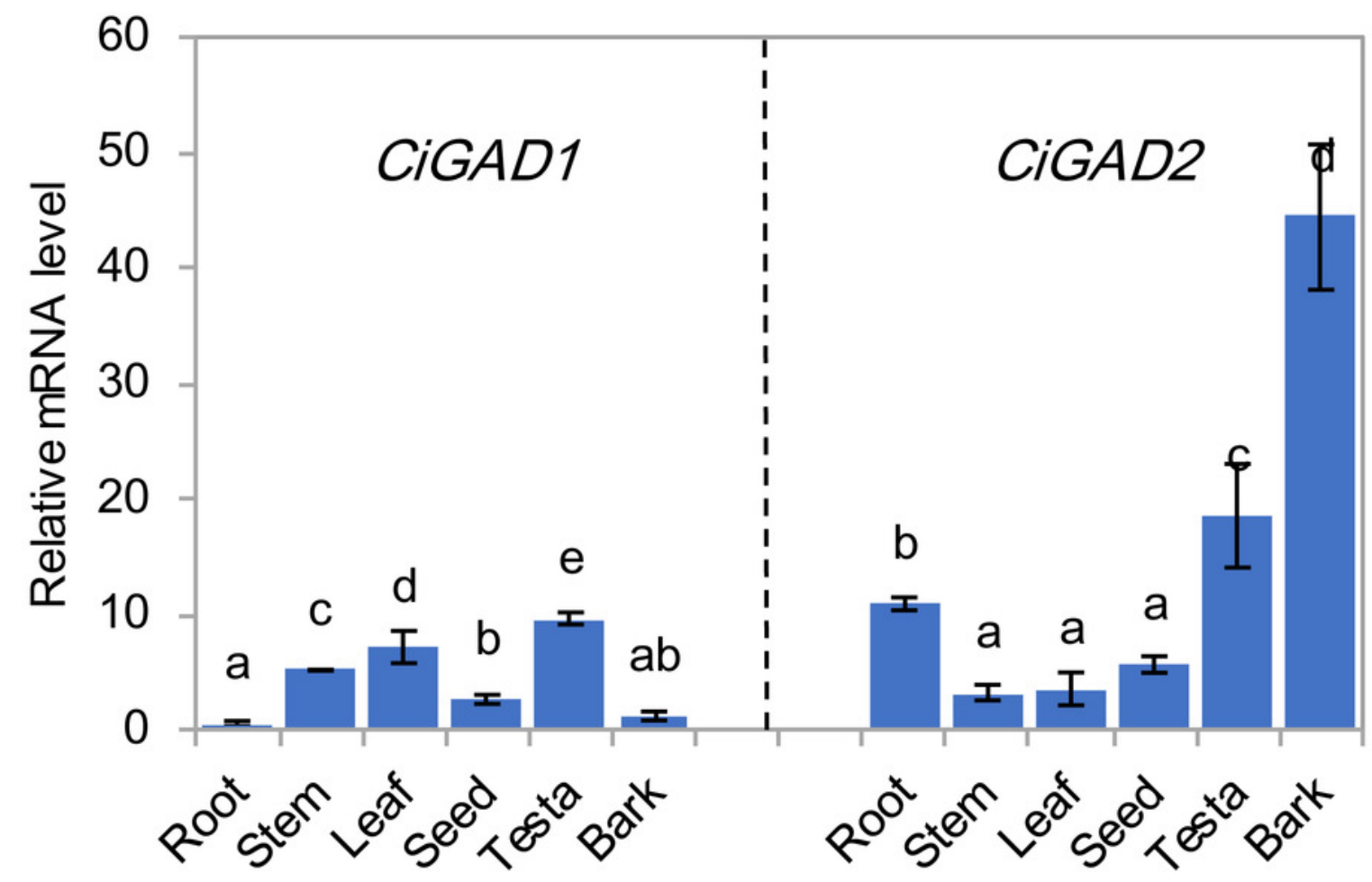

(B)

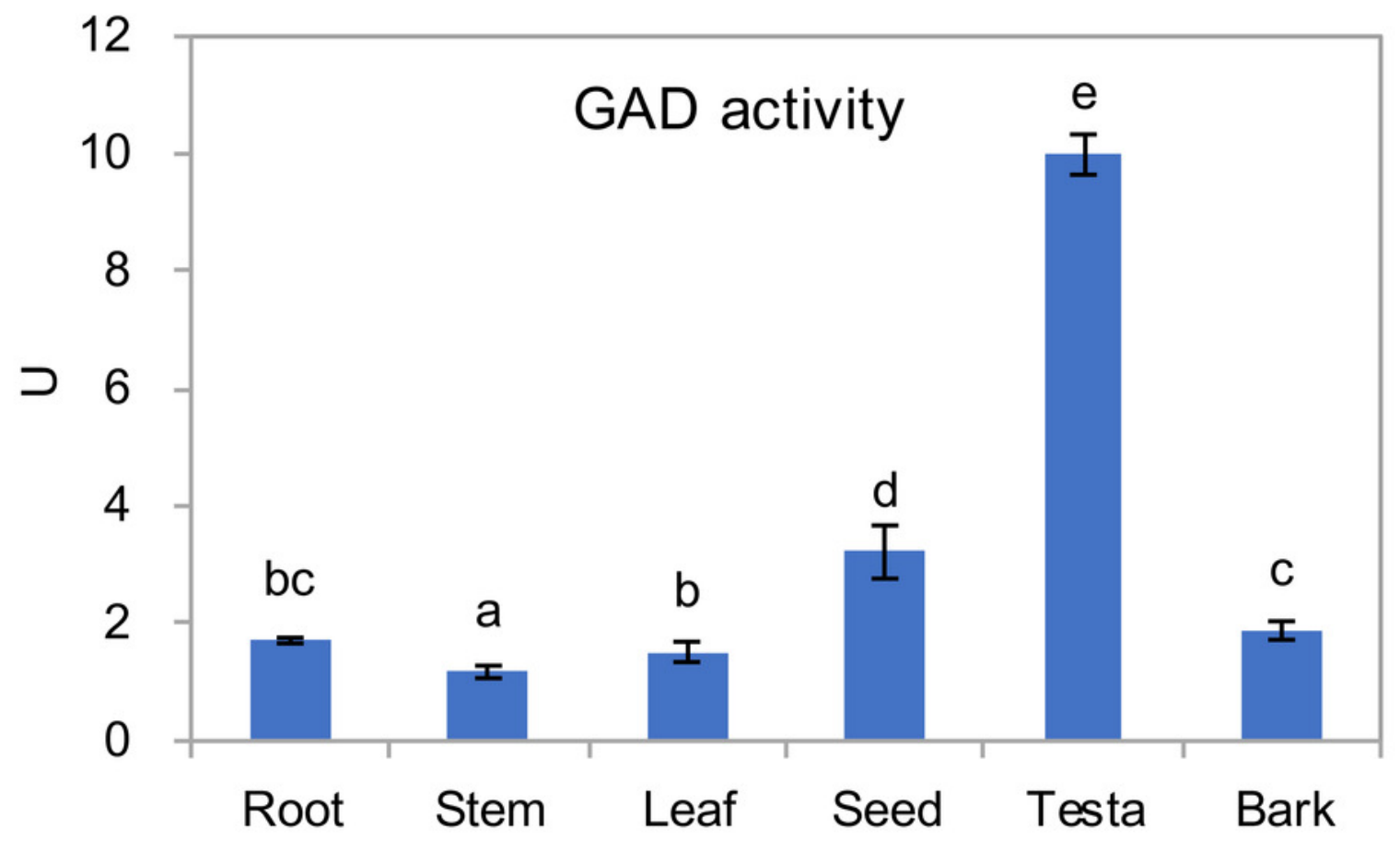




\section{Figure 5}

Stress-specific expression of two CiGADs in roots and leaves of young Caragana intermedia seedlings under different stress treatments for $3 \mathrm{~h}$.

CK: Water; Na: 200 mM NaCl; Zn: $100 \mu \mathrm{M} \mathrm{ZnSO}_{4} ; \mathrm{Cd}: 500 \mu \mathrm{M} \mathrm{CdCl}_{2} ; \mathrm{HT}$ : High temperature 40 ${ }^{\circ} \mathrm{C}$; LT: Low temperature $4{ }^{\circ} \mathrm{C}$; DE: Dehydration. The means and standard errors were calculated from three biological replicates. Different lower-case letters between any two sampling points indicate signification difference at $P<0.05$ by Duncan's test. 

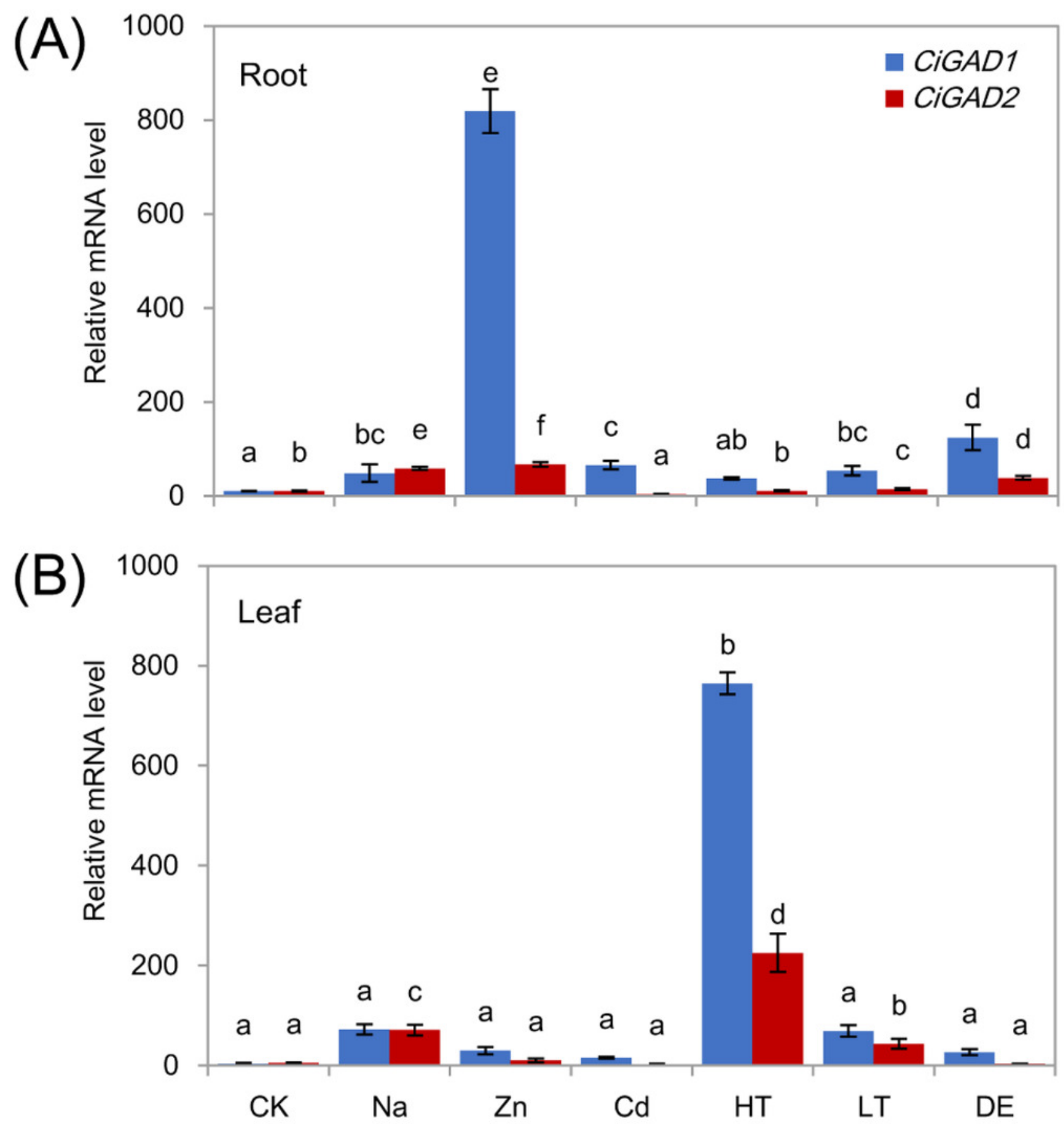


\section{Figure 6}

Expression of CiGADs and production of GABA in roots and leaves of young Caragana intermedia seedlings under $200 \mathrm{mM} \mathrm{NaCl}$ treatment for $0,3,6,12$ and $24 \mathrm{~h}$.

(A) and (B) CiGADs' expression in root and leaves, respectively; (C) GABA production. The means and standard errors were calculated from three biological replicates. Different lowercase letters between any two sampling points indicate signification difference at $P<0.05$ by Duncan's test. 

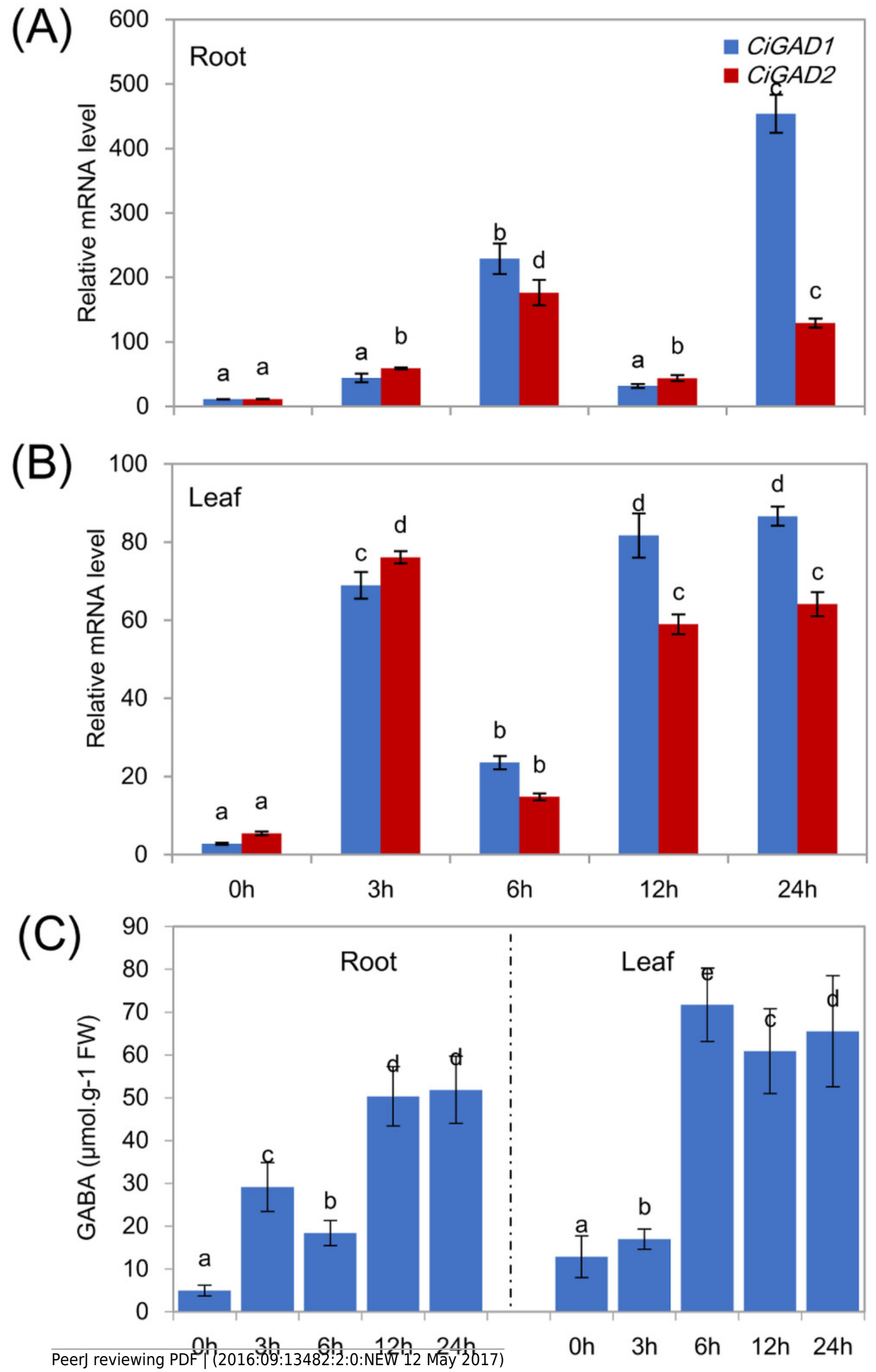


\section{Figure 7}

Effects of ABA on CiGADs' expression in roots (A) and leaves (B) of young Caragana intermedia seedlings under $200 \mathrm{mM} \mathrm{NaCl}$ treatment for 3 and $24 \mathrm{~h}$.

The concentration of exogenous ABA was $0,0.5,1,10,100$ and $200 \mu \mathrm{M}$. The means and standard errors were calculated from three biological replicates. Different lower-case letters between any two sampling points indicate signification difference at $P<0.05$ by Duncan's test. 

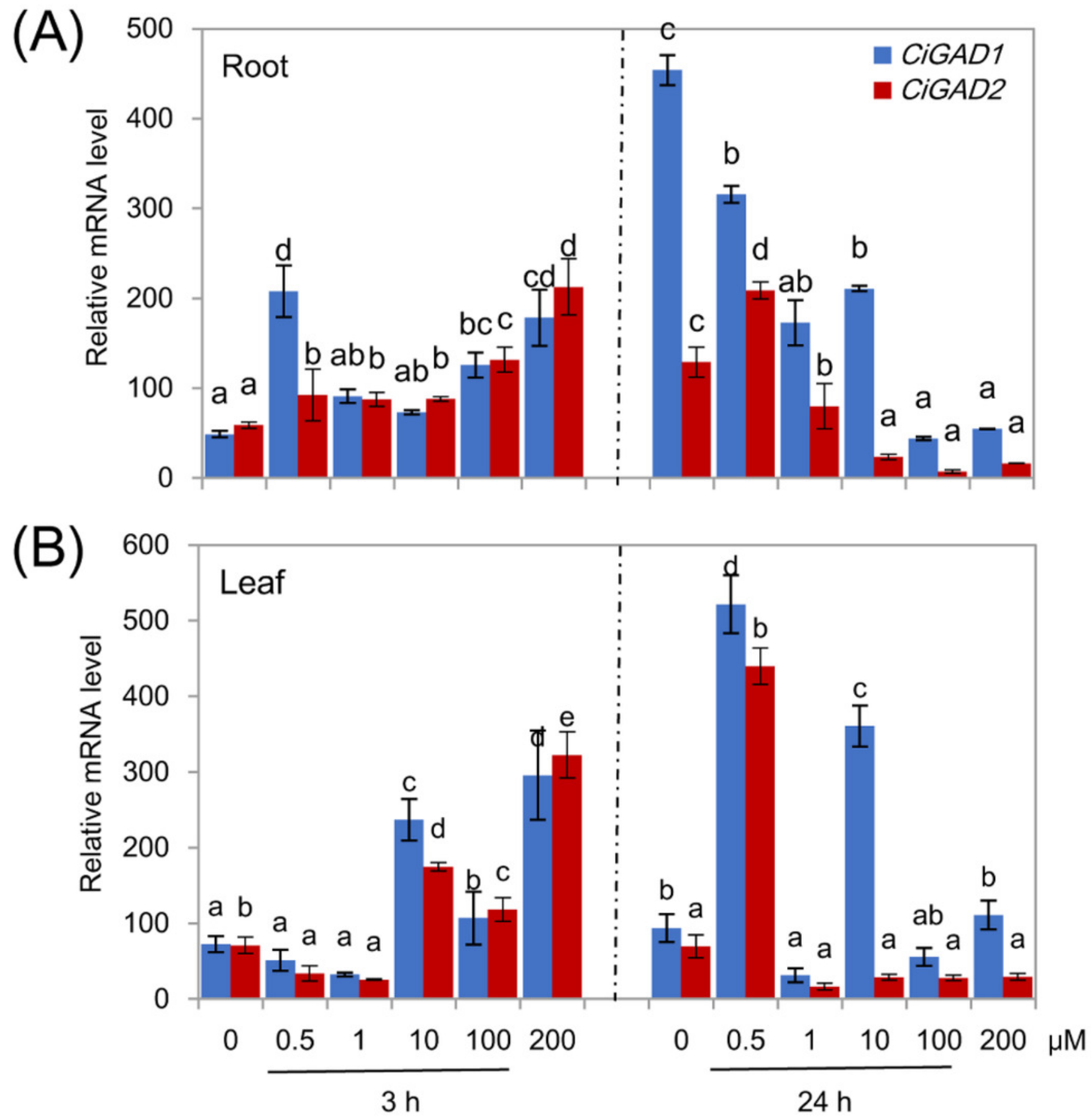